PROCEEDINGS OF THE AMERICAN MATHEMATICAL SOCIETY

Volume 128, Number 1, Pages 299-306

S 0002-9939(99)05193-X

Article electronically published on June 21, 1999

\title{
FREE ACTIONS OF FINITE GROUPS ON PRODUCTS OF SYMMETRIC POWERS OF EVEN SPHERES
}

\author{
SATYA DEO AND JITENDRA KUMAR MAITRA
}

(Communicated by Ralph Cohen)

\begin{abstract}
This paper answers a question on the existence of free actions on products of symmetric powers of even-spheres. The main objective is to show that a finite group $G$ acts freely on a finite product of symmetric powers of even-dimensional spheres iff it can act freely on a suitable product of evendimensional spheres themselves.
\end{abstract}

\section{INTRODUCTION}

Let $S^{2 d}, d \geq 1$, denote the $2 d$-dimensional sphere, and for any $h \geq 1$, let $S P^{h}\left(S^{2 d}\right)$ denote the orbit space of the Cartesian product $\prod_{1}^{h}\left(S^{2 d}\right)$ under the obvious action of the symmetric group of degree $h$. Then this orbit space $S P^{h}\left(S^{2 d}\right)$ is called the $h$-fold symmetric power of $S^{2 d}$ (see Dold [6], p. 60). In case $h=1$, it is obviously the sphere $S^{2 d}$ itself, and in case $h>1$ and $d=1, S P^{h}\left(S^{2}\right)$ is the complex projective space $C P^{h}$. In general, it is well known that its rational cohomology algebra $H^{*}\left(S P^{h}\left(S^{2 d}\right) ; Q\right)$ is a truncated polynomial algebra $Q[x] /\left(x^{h+1}\right)$ where the degree of $x$ is $2 d$ and the height of $x$ is $h$, in the sense that $x^{h} \neq 0$ but $x^{h+1}=0$ in $H^{*}\left(S P^{h}\left(S^{2 d}\right) ; Q\right)$ (see Dold [7]). As in the familiar case of the even-dimensional sphere $S^{2 d}$, it follows from the Lefschetz Fixed Point Theorem that the only finite group $G$ which can act freely on $S P^{h}\left(S^{2 d}\right)$ is $G \cong Z_{2}$; and in that case $h$ has to be an odd integer. Conversely, M. Hoffman [8] has shown that there is a free action of $G=Z_{2}$ on $S P^{h}\left(S^{2 d}\right)$ for any $h$-odd and any positive integer $d$.

Cusick [3], [4] has shown that if a finite group $G$ acts freely on $\prod_{1}^{m}\left(C P^{h_{i}}\right)^{s_{i}}$ or $\prod_{1}^{m}\left(S^{2 d_{i}}\right)^{s_{i}}$, then $G$ is a 2-group. Generalizing this result M. Hoffman [8] has shown that if $G$ is a finite group acting freely on an arbitrary product of symmetric powers of even-dimensional spheres, then $G$ is a 2-group. It appears that perhaps, different 2-groups can act freely on different symmetric powers of even-dimensional spheres depending on their degrees $2 d_{i}$ and heights $h_{i}$. In this paper we show that the class of finite groups which can act freely on any product of symmetric powers of even spheres is precisely the class of finite groups which can act freely on a suitable product of even-dimensional spheres.

Received by the editors July 29, 1997 and, in revised form, March 24, 1998.

1991 Mathematics Subject Classification. Primary 57S17; Secondary 57S05.

Key words and phrases. Lefschetz number, free action, symmetric powers, Wreath products.

While carrying out this work, the first author was supported by the UGC research grant no. F 8-5/94(SR-I) and the second author was supported by Dr. K.S. Krishnan Research Fellowship, awarded by the Department of Atomic Energy (No. 11/18/93-G), Govt. of India.

(C)1999 American Mathematical Society 
Let $X$ be a product of symmetric powers of even-dimensional spheres. First we note that such an arbitrary product will be of the form

$$
X=\prod_{i=1}^{m} \prod_{j=1}^{n_{i}}\left(S P^{h_{i j}}\left(S^{2 d_{i}}\right)\right)^{s_{i j}}
$$

where we allow $d_{i}$ to vary in strictly increasing order, and for each fixed $d_{i}$, we allow $h_{i j}$ to also vary in strictly increasing order: The powers $s_{i j}$ represent the number (or size) of generators of the rational cohomology algebra of $X$ which are of degree $2 d_{i}$ and height $h_{i j}$ and are given for every given pair $\left(h_{i j}, d_{i}\right)$ of positive integers. The special case of product of even-dimensional spheres (viz. when $h_{i j}=1$ ) will look like

$$
Y=\prod_{i=1}^{m}\left(S^{2 d_{i}}\right)^{s_{i}}
$$

whereas the case of product of complex projective spaces (viz. $d_{i}=1$ ) will look like

$$
Z=\prod_{i=1}^{m}\left(C P^{h_{i}}\right)^{s_{i}}
$$

With these notations, M. Hoffman's result says that if a finite group $G$ acts freely on a $\mathrm{CW}$-complex having the same homotopy type as $X$, then $G$ must be a 2-group. As mentioned earlier, the same result, for the special cases of CW-complex having the homotopy type of $Y$ and $Z$, was proved by L. Cusick [3], [4]. by

Given the space $X$, let $Y$ be the product of even-dimensional spheres, defined

$$
Y=\prod_{i=1}^{m} \prod_{j=1}^{n_{i}}\left(S^{2 d_{i} h_{i j}+\left(2 d_{1} h_{1 n_{1}}+\cdots+2 d_{i-1} h_{i-1 n_{i-1}}\right)}\right)^{s_{i j}},
$$

with the assumption that $2 d_{1} h_{1 n_{1}}+\cdots+2 d_{i-1} h_{i-1 n_{i-1}}=0$ if $i=1$. The main result of this paper (Theorem 3.1) will assert that a finite group $G$ acts freely on a CW-complex having the homotopy type of $X$ iff it acts freely on a CW-complex having the homotopy type of $Y$. In particular, the class of finite groups which can act freely on an arbitrary finite product of symmetric powers of even spheres is precisely the class of finite groups which can act freely on a suitable finite product of even-dimensional spheres. In other words, the apparent enlargement of the class of $\mathrm{CW}$-complexes by taking products of symmetric powers of even-dimensional spheres does not really enlarge the class of finite groups which can act freely on such spaces as compared to the class, which can act freely on only products of even-dimensional spheres.

The authors are thankful to the referee for suggesting some improvements in our exposition.

\section{Automorphism groups of RATionAl COHOMOLOGY ALGEBRAS ACTING ON A SPACE}

In this paper, we consider only finite (more generally, discrete) group actions on topological spaces unless stated otherwise. Let $X, Y$ be two topological spaces, and $H^{*}(X ; Q), H^{*}(Y ; Q)$ denote the rational cohomology algebras of $X$ and $Y$ respectively. Suppose $G^{\prime}$ is a discrete topological group acting on $Y$ and the group 
$\operatorname{Aut}\left(H^{*}(X ; Q)\right)$ of automorphisms of the rational cohomology algebra of $X$ is contained in $G^{\prime}$. Then for any discrete group $G$ which acts on $X$, there is an induced action of $G$ on $Y$ defined as follows:

$$
g . y=g^{*} \cdot y .
$$

Here $g^{*}$ is the automorphism of $H^{*}(X ; Q)$ induced by the homeomorphism $x \rightarrow g . x$ defined by the action of $G$ on $X$; the action on the right is the given action of $G^{\prime}$ on $Y$.

Proposition 2.1. Let $X, Y$ be two spaces. Suppose for some coefficient $\Lambda$, the groups Homeo $(X)$ and Homeo $(Y)$ have faithful induced representations in the automorphism groups $\operatorname{Aut}\left(H^{*}(X ; \Lambda)\right)$ and $\operatorname{Aut}\left(H^{*}(Y ; \Lambda)\right)$, each of which is isomorphic to a group $G^{\prime}$ which acts effectively (resp. freely) on $X$ as well as $Y$. Then a group $G$ can act effectively (resp. freely) on $X$ iff it can act effectively (resp. freely) on $Y$.

Proof. Case I. Suppose $G$ acts effectively on $X$. Then the induced homomorphim $\rho: G \rightarrow H o m e o(X)$ is injective. This implies, by our hypothesis, that the induced homomorphism $\rho^{\prime}: G \rightarrow \operatorname{Aut}\left(H^{*}(X ; \Lambda)\right)=G^{\prime}$ is an injection. Since $G^{\prime}$ acts on $Y$ effectively, the induced homomorphism $\rho^{\prime \prime}: G^{\prime} \rightarrow H o m e o(Y)$ is also injective. This means that the composite homomorphism $\rho^{\prime \prime} \circ \rho^{\prime}: G \rightarrow H o m e o(Y)$ is injective, and so equation (1) defines an effective action of $G$ on $Y$. The converse is now similarly obvious.

Case II. Suppose $G$ acts freely on $X$. Since $G^{\prime}$ acts freely on $Y$, every nonidentity element of $G^{\prime}$ moves every point of $Y$. Since $G$ acts freely on $X$, the homomorphism $\rho^{\prime}: G \rightarrow G^{\prime}$ is a faithful representation of $G$ in $G^{\prime}$, and so it follows that every nonidentity element of $G$ also acts freely on $Y$. The converse is now similarly obvious.

The proof of the following is now evident and hence omitted.

Proposition 2.2. Suppose $X$ and $Y$ are two spaces. For some coefficient $\Lambda$, suppose the groups $H_{o m e o}(X)$ and Homeo $(Y)$ have faithful induced representations in the automorphism groups Aut $\left(H^{*}(X ; \Lambda)\right)$ and Aut $\left(H^{*}(Y ; \Lambda)\right)$ each of which is isomorphic to a group $G^{\prime}$, which acts on $X$ and $Y$ both. Let $G$ be a finite group which acts on spaces $X$ and $Y$ and let $\rho: G \rightarrow G^{\prime}$ be the induced representation. Suppose $S$ is a subset of $G^{\prime}$ satisfying the condition that if $g \in G$ acts freely on $X$ or $Y$, then $\rho(g) \in S$ and every element of $S$ acts freely on $X$ and $Y$ under the induced action from $G^{\prime}$. Then $G$ acts on $X$ freely iff it acts on $Y$ freely.

Recall that if $G$ is a finite group, then the symmetric group $\sum_{n}$ of degree $n$ acts on the $n$-fold direct product group $\prod_{1}^{n} G$. The semidirect product of $G^{n}$ and $\sum_{n}$ is denoted by $\sum_{n} \int G$ and is called the $n$-fold Wreath product of $G$ by itself. Let $G$ act on a space $X$ freely. Then the Wreath product $\sum_{n} \int G$ acts on the product space $X^{n}$ by the action

$$
\left(g_{1}, \ldots, g_{n} ; \sigma\right) \cdot\left(x_{1}, \ldots, x_{n}\right)=\left(g_{1} \cdot x_{\sigma^{-1}(1)}, \ldots, g_{n} \cdot x_{\sigma^{-1}(n)}\right) .
$$

More generally, if a finite group $G$ acts freely on the spaces $X_{1}, \ldots, X_{k}$ and $n_{1}, \ldots, n_{k}$ are positive integers, then the direct product group $\sum_{n_{1}} \int G \times \cdots \times$ $\sum_{n_{k}} \int G$ acts on the product space $X_{1}^{n_{1}} \times \cdots \times X_{k}^{n_{k}}$ by the product action.

It is easily seen that even if $G$ acts on $X$ freely, the Wreath product $\sum_{n} \int G$ need not act freely on $X^{n}$, but some elements of $\sum_{n} \int G$ can act freely on $X^{n}$, i.e., can 
move every element of $X^{n}$. To determine such elements we require the following definition.

Definition 2.1. We say that an element $\left(g_{1}, \ldots, g_{n} ; \sigma\right) \in \sum_{n} \int G$ satisfies the cycle condition if for some cycle $\left(i, \sigma(i), \ldots, \sigma^{l-1}(i)\right)$ of $\sigma$,

$$
g_{i} \cdot g_{\sigma^{-1}(i)} \cdots \cdot g_{\sigma^{1-l}(i)} \neq i d e n t i t y .
$$

Observe that if groups $G_{1}, \ldots, G_{n}$ act on spaces $X_{1}, \ldots, X_{n}$ respectively, then the product group $G_{1} \times \cdots \times G_{n}$ acts on the product space $X_{1} \times \cdots \times X_{n}$ under the product action. Also note that an element $\left(g_{1}, \ldots, g_{n}\right)$ moves every point of $X_{1} \times \cdots \times X_{n}$ iff there exists a $g_{i}$ which moves every element of $X_{i}$ : to see this note that if $g_{i}$ moves every point of $X_{i}$, then clearly $\left(g_{1}, \ldots, g_{i}, \ldots, g_{n}\right)$ will move any point $\left(x_{1}, \ldots, x_{i}, \ldots, x_{n}\right)$ of the product because $x_{i} \neq g_{i} . x_{i}$. Conversely suppose there is no such $g_{i}$. This means for all $j=1, \ldots, n, \exists a_{j} \in X_{j}$ such that $g_{j} . a_{j}=a_{j}$. But then, by definition, $\left(g_{1}, \ldots, g_{n}\right) \cdot\left(a_{1}, \ldots, a_{n}\right)=\left(a_{1}, \ldots, a_{n}\right)$, a contradiction to the fact that $\left(g_{1}, \ldots, g_{n}\right)$ moves every point of the product.

Now we consider the product group

$$
\sum_{n_{1}} \int G \times \cdots \times \sum_{n_{k}} \int G
$$

where $n_{1}, \ldots, n_{k}$ are any $k$ positive integers. Since all of the symmetric groups $\sum_{n_{1}}, \ldots, \sum_{n_{k}}$ can be considered as subgroups of the symmetric group $\sum_{n}$ where $n=n_{1}+\cdots+n_{k}$ (by making symbols disjoint), an element

$$
\left(\left(g_{1}, \ldots, g_{n_{1}} ; \sigma_{n_{1}}\right), \ldots,\left(h_{1}, \ldots, h_{n_{k}} ; \sigma_{n_{k}}\right)\right)
$$

of the product group can really be considered as

$$
\left(\left(g_{1} \cdots g_{n_{1}}\right), \ldots,\left(h_{1} \cdots h_{n_{k}}\right) ; \sigma_{n_{1}} \circ \cdots \circ \sigma_{n_{k}}\right)
$$

which is an element of $\sum_{n} \int\left(G_{1} \times \cdots \times G_{k}\right)$. Note that $\sigma_{n_{1}}, \ldots, \sigma_{n_{k}}$ all commute among themselves. Now to say that the above element satisfies a cycle condition really means there exists a cycle of $\sum_{n}$ (and hence a cycle of $\sum_{n_{i}}$ for some $i$ ) such that the product of required elements of the group $G$ for the cycle condition is not the identity element of $G_{i}$. This, in turn, will mean that $G_{i}$ satisfies the cycle condition. Conversely, if $\sum_{n_{i}} \int G_{i}$ satisfies the cycle condition for some $i$, then evidently, the above product group $\prod \sum_{n_{i}} \int G_{i}$ also satisfies the cycle condition.

Now we prove the following known (cf. [8, p. 380]) general result in which we characterize the elements of the direct product $\sum_{n_{1}} \int G \times \cdots \times \sum_{n_{k}} \int G$ which can act freely on $X_{1}^{n_{1}} \times \cdots \times X_{k}^{n_{k}}$, in terms of the cycle condition.

Theorem 2.1. Suppose $G$ acts freely on each of the spaces $X_{1}, \ldots, X_{k}$ and let $n_{1}, \ldots, n_{k}$ be positive integers. Then a subgroup of the direct product $\sum_{n_{1}} \int G \times$ $\cdots \times \sum_{n_{k}} \int G$ acts freely on $X_{1}^{n_{1}} \times \cdots \times X_{k}^{n_{k}}$ iff all its nonidentity elements satisfy the cycle condition.

Proof. Let $S$ be a subgroup of the direct product group $\sum_{n_{1}} \int G \times \cdots \times \sum_{n_{k}} \int G$ and $\gamma=\left(\gamma_{1}, \ldots, \gamma_{k}\right) \in S$ be a nonidentity element of the group $S$ satisfying the cycle condition. This means there exists a $j, 1 \leq j \leq k$, such that $\gamma_{j} \in \sum_{n_{j}} \int G$ satisfies the cycle condition. We show that $\gamma_{j}$ moves every point of the space $X_{j}^{n_{j}}$. If possible suppose $\gamma_{j}=\left(g_{1}, \ldots, g_{n_{j}} ; \sigma_{j}\right) \in \sum_{n_{j}} \int G$ fixes some $\left(x_{1}, \ldots, x_{n_{j}}\right) \in X_{j}^{n_{j}}$, i.e.,

$$
\left(g_{1}, \ldots, g_{n_{j}} ; \sigma_{j}\right) .\left(x_{1}, \ldots, x_{n_{j}}\right)=\left(x_{1}, \ldots, x_{n_{j}}\right) .
$$


This implies

$$
\left(g_{1} \cdot x_{\sigma_{j}^{-1}(1)}, \ldots, g_{n_{j}} \cdot x_{\sigma_{j}^{-1}\left(n_{j}\right)}\right)=\left(x_{1}, \ldots, x_{n_{j}}\right) .
$$

If $\sigma_{j}$ is a cycle of length $l$, satisfying the cycle condition, then we find that

$$
x_{i}=g_{i} \cdot x_{\sigma_{j}^{-1}(i)}=\cdots=g_{i} g_{\sigma_{j}^{-1}(i)} \cdots g_{\sigma_{j}^{1-l}(i)} \cdot x_{i} .
$$

This shows that $g_{i} g_{\sigma_{j}^{-1}(i)} \cdots g_{\sigma_{j}^{1-l}(i)}=i d e n t i t y$, a contradiction. Thus $\gamma_{j}$ acts freely on $X_{j}^{n_{j}}$ and hence $\gamma=\left(\gamma_{1}, \ldots, \gamma_{k}\right)$ acts freely on $X$.

Conversely suppose $S$ is a subgroup of the direct product $\sum_{n_{1}} \int G \times \cdots \times \sum_{n_{k}} \int G$ which acts freely on $X$, and $\gamma=\left(\gamma_{1}, \ldots, \gamma_{k}\right)$ is any nonidentity element of the group $S$. Then we show that $\gamma$ satisfies the cycle condition. For this we have to show that for some $j, 1 \leq j \leq k, \gamma_{j}$ satisfies the cycle condition. If possible, suppose for all $j, 1 \leq j \leq k, \gamma_{j}=\left(g_{1}, \ldots, g_{n_{j}} ; \sigma_{j}\right) \in \sum_{n_{j}} \int G$ does not satisfy the cycle condition. This means for every cycle $\left(i, \sigma_{j}(i), \ldots, \sigma_{j}^{l-1}(i)\right)$ of $\sigma_{j}$, we have

$$
g_{i} g_{\sigma_{j}^{-1}(i)} \cdots g_{\sigma_{j}^{1-l}(i)}=\text { identity. }
$$

Now we choose $x_{0} \in X_{j}$ and define an element $\left(x_{1}, \ldots, x_{n_{j}}\right) \in X_{j}^{n_{j}}$ by $x_{i}=$ $x_{0}, x_{\sigma_{j}(i)}=g_{\sigma_{j}(i)} \cdot x_{0}, x_{\sigma_{j}^{2}(i)}=g_{\sigma_{j}^{2}(i)} g_{\sigma_{j}(i)} \cdot x_{0}, \ldots$, and so on, for every cycle $\left(i, \sigma_{j}(i), \ldots, \sigma_{j}^{l-1}(i)\right)$ of $\sigma_{j}$. Then from equation (2), we find that $g_{i} . x_{\sigma_{j}^{-1}(i)}=x_{i}$, i.e., $x_{j}=\left(x_{1}, \ldots, x_{n_{j}}\right) \in X_{j}^{n_{j}}$ is a fixed point of $\gamma_{j}=\left(g_{1}, \ldots, g_{n_{j}} ; \sigma_{j}\right)$. For each $j$, we choose such a point $x_{j} \in X_{j}^{n_{j}}$ such that $\gamma_{j} \cdot x_{j}=x_{j}$. This shows that $x=\left(x_{1}, \ldots, x_{k}\right) \in X$ is a fixed point of $\gamma=\left(\gamma_{1}, \ldots, \gamma_{k}\right) \in S$, a contradiction to the free action of $S$ on $X$.

\section{Free ACtions on PRoduCts of SyMmetric POWERS OF EVEN SPHERES}

Let us first consider the two product spaces

$$
X=\prod_{i=1}^{m} \prod_{j=1}^{n_{i}}\left(S P^{h_{i j}}\left(S^{2 d_{i}}\right)\right)^{s_{i j}}
$$

and

$$
X^{\prime}=\prod_{i=1}^{m} \prod_{j=1}^{n_{i}}\left(S P^{h_{i j}^{\prime}}\left(S^{2 d_{i}^{\prime}}\right)\right)^{s_{i j}},
$$

where without loss of generality we can assume that both $d_{i}$ and $d_{i}^{\prime}$ are arranged in strictly increasing order, and for each given $d_{i}\left(\operatorname{resp} d_{i}^{\prime}\right), h_{i j}\left(\operatorname{resp} h_{i j}^{\prime}\right)$ are also arranged in strictly increasing order. Note that powers $s_{i j}$ for each symmetric product remain the same. Obviously the rational cohomology algebras of both $X$ and $X^{\prime}$ need not be isomorphic since they depend on the degrees $2 d_{i}$ as well as heights $h_{i j}$ of symmetric powers. However, we have:

Proposition 3.1. Given the two spaces $X$ and $X^{\prime}$ as above, the group of automorphisms of their rational cohomology algebras are isomorphic.

Proof. Recall that for a given integer $n, \Sigma_{n}$ denotes the symmetric group of degree $n$, and $\Sigma_{n} \int Z_{2}$ denotes the $n$-fold Wreath product of the multiplicative group $Z_{2}$. If

$$
Q\left[x_{1}, \ldots, x_{n}\right] /\left(x_{1}^{h}, x_{2}^{h}, \ldots ., x_{n}^{h}\right)
$$


is a truncated rational polynomial algebra where degrees of each generator $x_{i}$ are $d$ and heights are $h$, then any mapping which takes an $x_{i}$ to $a_{i} x_{\sigma(i)}$, where $\sigma \in \Sigma_{n}$ and $a_{i} \in Q^{*}$, the set of nonzero elements of $Q$, will evidently define an automorphism of the above truncated polynomial algebra. Since automorphisms of the above algebra cannot change the degrees of elements, and are themselves completely determined by generators, the converse is also easily verified [5]. Therefore, it should now be clear that an automorphism of the above truncated polynomial algebra is independent of the degree as well as height of the generators.

Note that the rational cohomology algebra of the space $X$ is simply the tensor product of the rational cohomology algebras of the symmetric powers viz. $X_{i j}=$ $\left(S P^{h_{i j}}\left(S^{2 d_{i}}\right)\right)^{s_{i j}}$. We know that the rational cohomology algebra of the space $X_{i j}$ is a truncated polynomial algebra on $s_{i j}$ generators each of degree $2 d_{i}$ and height $h_{i j}$, i.e.,

$$
H^{*}\left(X_{i j} ; Q\right)=Q\left[x_{1}, \ldots, x_{s_{i j}}\right] /\left(x_{1}^{h_{i j}}, \ldots, x_{s_{i j}}^{h_{i j}}\right) .
$$

Therefore, in view of our beginning remarks, the group of automorphisms of $H^{*}\left(X_{i j} ; Q\right)$ is isomorphic to the Wreath product $\sum_{s_{i j}} \int Q^{*}$ which is independent of the degree $2 d_{i}$ and height $h_{i j}$. Also, if $A$ and $B$ are two $Q$-algebras and every automorphism of their tensor product maps elements of $A$ into $A$ and elements of $B$ into $B$, then clearly

$$
A u t(A \otimes B) \simeq A u t(A) \times A u t(B) .
$$

It follows, therefore, that

$$
\begin{aligned}
\operatorname{Aut}\left(H^{*}(X ; Q)\right) & \cong \prod_{i=1}^{m} \prod_{j=1}^{n_{i}} \operatorname{Aut}\left(H^{*}\left(X_{i j} ; Q\right)\right) \\
& =\prod_{i=1}^{m} \prod_{j=1}^{n_{i}}\left(\sum_{s_{i j}} \int Q^{*}\right) .
\end{aligned}
$$

By a similar argument, we have

$$
\operatorname{Aut}\left(H^{*}\left(X^{\prime} ; Q\right)\right) \cong \prod_{i=1}^{m} \prod_{j=1}^{n_{i}}\left(\sum_{s_{i j}} \int Q^{*}\right),
$$

which completes the proof.

Let us denote the direct product group $\prod_{i=1}^{m} \prod_{j=1}^{n_{i}} \sum_{s_{i j}} \int Z_{2}$ by $\mathcal{G}$. Then we note that the group $\mathcal{G}$ being the direct product of a finite number of finite groups is finite and is a subgroup of the above automorphism group because $Z_{2} \subseteq Q^{*}$. Next, we have:

Proposition 3.2. Let

$$
X=\prod_{i=1}^{m} \prod_{j=1}^{n_{i}}\left(S P^{h_{i j}}\left(S^{2 d_{i}}\right)\right)^{s_{i j}}
$$

and

$$
X^{\prime}=\prod_{i=1}^{m} \prod_{j=1}^{n_{i}}\left(S P^{h_{i j}^{\prime}}\left(S^{2 d_{i}^{\prime}}\right)\right)^{s_{i j}}
$$


be two spaces. If $h_{i j}$ and $h_{i j}^{\prime}$ are odd for all $i$ and $j$, then there is a natural action of the finite group $\mathcal{G}$ on $X$ and $X^{\prime}$ both induced by Hoffman's free action of $Z_{2}$ on $S P^{h_{i j}}\left(S^{2 d_{i}}\right)$ and $S P^{h_{i j}^{\prime}}\left(S^{2 d_{i}^{\prime}}\right)$ respectively.

Proof. For each pair $(i, j)$ since $h_{i j}$ is odd, there is a free action of $Z_{2}$ on $S P^{h_{i j}}\left(S^{2 d_{i}}\right)$ (see Hoffman [8], p. 385). Consequently, for every positive integer $s_{i j}$ the group $\sum_{s_{i j}} \int Z_{2}$ acts on the product space $\left(S P^{h_{i j}}\left(S^{2 d_{i}}\right)\right)^{s_{i j}}$ by the action

$$
\left(a_{1}, \ldots, a_{s_{i j}} ; \sigma\right) \cdot\left(x_{1}, \ldots, x_{s_{i j}}\right)=\left(a_{1} \cdot x_{\sigma^{-1}(1)}, \ldots, a_{s_{i j}} \cdot x_{\sigma^{-1}\left(s_{i j}\right)}\right) .
$$

But then the group $\mathcal{G}$ acts on the product space $X=\prod_{i=1}^{m} \prod_{j=1}^{n_{i}}\left(S P^{h_{i j}}\left(S^{2 d_{i}}\right)\right)^{s_{i j}}$ by the product action.

A similar argument gives an action of the group $\mathcal{G}$ on $X^{\prime}$.

Next we have:

Proposition 3.3. Let $X$ and $X^{\prime}$ be the two spaces as defined above. Then a finite group $G$ acts on $X$ freely iff it acts on $X^{\prime}$ freely.

Proof. By Proposition 3.1 first we note that the automorphism group of the rational cohomology algebras of $X$ and $X^{\prime}$ are isomorphic. In fact

$$
\operatorname{Aut}\left(H^{*}(X ; Q)\right) \cong \operatorname{Aut}\left(H^{*}\left(X^{\prime} ; Q\right)\right) \supset \prod_{i=1}^{m} \prod_{j=1}^{n_{i}}\left(\sum_{s_{i j}} \int Z_{2}\right)=\mathcal{G} .
$$

From Proposition 3.2, it is clear that the group $\mathcal{G}$ acts on both the spaces $X$ and $X^{\prime}$. Let $S$ be the set of elements in $\mathcal{G}$ which satisfy the cycle condition. Now suppose $G$ acts freely on $X$. Let $\rho: G \rightarrow \mathcal{G}$ be the representation of $G$ in $\mathcal{G}$. For any $g(\neq e) \in G$, assume that $\rho(g)=\prod_{i=1}^{m} \prod_{j=1}^{n_{i}}\left(a_{1}, \ldots, a_{s_{i j}} ; \sigma_{s_{i j}}\right)$. Since $G$ acts freely on $X$, the Lefschetz number $L\left(g^{*}\right)$ of the automorphism $g^{*}: H^{*}(X ; Q) \rightarrow$ $H^{*}(X ; Q)$ must vanish, i.e., $L\left(g^{*}\right)=0$. Then from Theorem 3.2 of Hoffman [8] it follows that for some $i, j$ we have $a_{1} \cdots a_{s_{i j}}=-1$. This means that the element $\left(a_{1}, \ldots, a_{s_{i j}} ; \sigma_{s_{i j}}\right) \in \mathcal{G}$ satisfies the cycle condition, which implies that $\rho(g)$ in $\mathcal{G}$ satisfies the cycle condition and so $\rho(g) \in S$ and also $\rho$ is faithful. Since every element of $S$ satisfies the cycle condition, from Theorem 2.1, it follows that every element of $S$ acts freely on $X$ and $X^{\prime}$ under the induced action of $\mathcal{G}$. Therefore from Proposition 2.2, $G$ acts freely on $X^{\prime}$.

By a similar argument we can prove that, if $G$ acts freely on $X^{\prime}$, then it acts freely on $X$.

Finally we have our main result:

Theorem 3.1. Let

$$
X=\prod_{i=1}^{m} \prod_{j=1}^{n_{i}}\left(S P^{h_{i j}}\left(S^{2 d_{i}}\right)\right)^{s_{i j}}
$$

and

$$
Y=\prod_{i=1}^{m} \prod_{j=1}^{n_{i}}\left(S^{2 d_{i} h_{i j}+\left(2 d_{1} h_{1 n_{1}}+\cdots+2 d_{i-1} h_{i-1 n_{i-1}}\right)}\right)^{s_{i j}}
$$

be two spaces, where the $h_{i j}$ 's are all odd. Then a finite group $G$ acts on $X$ freely iff it acts on $Y$ freely. 
Proof. First we observe that the rational cohomology algebra of the space $Y$ is a tensor product of the truncated polynomial algebra on $s_{i j}$ generators of degree $2 d_{i} h_{i j}+\left(2 d_{1} h_{1 n_{1}}+\cdots+2 d_{i-1} h_{i-1 n_{i-1}}\right)$ and of height 1 . Hence as explained in the proof of Proposition 3.1, we have

$$
\operatorname{Aut}\left(H^{*}(Y ; Q)\right) \cong \prod_{i=1}^{m} \prod_{j=1}^{n_{i}}\left(\sum_{s_{i j}} \int Q^{*}\right) .
$$

Also

$$
\operatorname{Aut}\left(H^{*}(X ; Q)\right) \cong \operatorname{Aut}\left(H^{*}(Y ; Q)\right) \supset \mathcal{G} .
$$

Since $Z_{2}$ acts freely on any even-dimensional sphere, the group $\mathcal{G}$ acts on the space $Y$. Let $S$ be the set of elements in $\mathcal{G}$ satisfying the cycle condition. Then from Theorem 2.1, every element of $S$ acts freely on both the spaces $X$ and $Y$ under the induced action from $\mathcal{G}$.

Now suppose $G$ acts freely on $X$ or $Y$. Let $\rho: G \rightarrow \mathcal{G}$ be the representation of $G$ in $\mathcal{G}$. Then for any nonidentity element $g \in G$, the Lefschetz number of the induced automorphism $g^{*}$ must be zero. From this it follows that $\rho(g)$ in $\mathcal{G}$ satisfies the cycle condition and the representation $\rho$ is faithful. Thus we find that $\rho(G) \subset S$. Hence by Proposition 3.3, we find that $G$ acts freely on $X$ iff it acts freely on $Y$.

Corollary 3.1. Let $X$ be the product of odd-dimensional complex projective spaces, i.e.,

$$
X=\left(C P^{h_{1}}\right)^{s_{1}} \times \cdots \times\left(C P^{h_{m}}\right)^{s_{m}}
$$

where the $h_{i}$ 's are all odd integers and $Y$ is the product of even-dimensional spheres, viz.

$$
Y=\left(S^{2 h_{1}}\right)^{s_{1}} \times \cdots \times\left(S^{2 h_{m}}\right)^{s_{m}} .
$$

Suppose $G$ is a finite group. Then $G$ acts freely on $X$, iff it acts freely on $Y$.

\section{REFERENCES}

[1] A. Adem and R.J.Milgram, "Cohomology of Finite Groups", Springer-Verlag, New York (1994). MR 96f:20082

[2] G.E.Bredon, "Introduction to Compact Transformation Groups", Academic Press, New York (1972). MR 54:1265

[3] L.W.Cusick, "Free actions on products of even dimensional spheres", Proc. Amer. Math. Soc. 99 (1987), 573-574. MR 88h:57035

[4] L.W.Cusick, "Free actions on spaces with nonzero Euler characteristic", Topology and its Applications 33 (1989), 185-196. MR 90j:57033

[5] Satya Deo and J.K. Maitra, "Automorphism groups of truncated polynomial algebras", National Acad. Sciences Letters 20(1997)75-80.

[6] A. Dold, "Fixed-point properties of product spaces", Russian Math. Survey 31 (1976), 56-61. MR 57:1477

[7] A. Dold, "Homology of symmetric products and other functors of complexes", Ann. of Math. 68 (1958), 54-80. MR 20:3537

[8] M. Hoffman, "Homological restrictions on free group actions", Indiana Univ. Math. J. 37 (1988), 375-387. MR 89k:57079

[9] J. Milnor, "Groups which act on $S^{n}$ without fixed points", Amer. J. Math. 79 (1957), 623-630. MR 19:761d

[10] E.H.Spanier, "Algebraic Topology", Mc-Graw Hill, New York (1966). MR 35:1007

Department of Mathematics and Computer Science, R.D. University, Jabalpur - 482 001, INDIA

E-mail address: sdt@rdunijb.ren.nic.in

E-mail address: maitra@rdunijb.ren.nic.in 